\title{
Mensuração da velocidade de deslocamento de corpos rígidos em tempo real por análise de sequência de imagens e pela transformação empírica baseada nas funções polinomiais
}

\author{
Danilo Filitto ${ }^{1}$ \\ Airton Marco Polidorio ${ }^{2}$ \\ Júlio Kiyoshi Hasegawa ${ }^{3}$ \\ Nardênio Almeida Martins \\ Franklin César Flores ${ }^{1}$
}

\begin{abstract}
Resumo: Este trabalho apresenta uma metodologia para mensurar a velocidade de um corpo rígido em movimento por meio da análise de sequências de imagens adquiridas por uma filmadora digital comercial. Tal metodologia necessita do resultado da segmentação dos corpos de interesse em movimento nas sequências de imagens. Esse resultado é obtido pela aplicação da técnica de segmentação baseada na média temporal dos valores de brilho de cada pixel registrado em $N$ quadros de imagens consecutivos. Após a detecção e a segmentação dos corpos em movimento, alguns pontos dispostos sobre as suas imagens (pontos do espaço-imagem) são transformados em coordenadas métricas do espaço-objeto. Neste trabalho, essa transformação é feita pela aplicação do modelo matemático obtido por transformações feitas pelo uso das funções polinomiais. Transformando os pontos desejados do plano-imagem (2D) para seus respectivos correspondentes no espaço-objeto (3D), a distância percorrida pelo corpo em um determinado intervalo de tempo pode ser calculada e, dessa forma, a velocidade de deslocamento do corpo é mensurada. Para validar a metodologia proposta, foi montado um experimento, no qual um corpo desliza sobre um trilho de ar (para minimizar efeito do atrito), passando por cinco sensores fotoelétricos conectados a um cronômetro. No momento em que o corpo passa por um sensor, o respectivo cronômetro é paralisado e fornece o valor do intervalo de tempo (com referência ao primeiro sensor). As distâncias entre os sensores e os intervalos de tempo gastos para cruzar cada sensor permitem calcular a velocidade média de deslocamento do corpo com precisão de $\pm 1 \%$. A partir disso, tomase esses valores como corretos, os quais podem ser usados numa comparação com a velocidade calculada somente com base na análise de sequência de imagens.
\end{abstract}

Palavras-chave: Polinômios racionais. Segmentação. Transformação geométrica. Velocidade.

\begin{abstract}
This paper presents a methodology to measure the velocity of a rigid body in motion by analyzing image sequences acquired by a commercial digital camcorder. This methodology requires the segmentation result of the body motion of interest in image sequences. This result is obtained by applying the segmentation technique based on the temporal average of the brightness values of each pixel recorded in $N$ consecutive image frames. Following the detection and segmentation of moving bodies, some points arranged on the images of these bodies (the image space points) are transformed into object space coordinates metrics. In this work, this transformation is done by applying the mathematical model obtained by means of the transformations made by the use of polynomial functions. Transformed into the desired points of the image plane (2D) to their corresponding object in space (3D), the distance traveled by the body in a given time interval can be calculated and thus the velocity of displacement of the body is measured. To validate the proposed method, an experiment was installed in a body which slides on a rail air (to minimize the effect of friction) through five photoelectric sensors connected to a stopwatch. At the time when the body passes a sensor, its timer is stopped and provides the value
\end{abstract}

\footnotetext{
${ }^{1}$ Curso de Pós-Graduação em Ciência da Computação, UEM -Maringá (PR) - Brasil

\{dfilitto@gmail.com; nardenio, fcflores@din.uem.br\}

${ }^{2}$ Departamento de Informática - Universidade Estadual de Maringá

\{ampolidorio@gmail.com\}

${ }^{3}$ Departamento de Cartografia, UNESP - Faculdade de Ciências e Tecnologia -Presidente Prudente (SP) - Brasil

$\{$ hasegawalfct.unesp.br\}
}

http://dx.doi.org/10.5335/rbca.2014.2857 
of time interval that the body has led to pass by that sensor (with reference to the first sensor). The distances between the sensors and the intervals of time spent to cross each sensor can calculate the average velocity of the body with accuracy of $\pm 1 \%$. It becomes then these values as correct and that can be used in comparison with the calculated velocity with only the image sequence analysis.

Keywords: Image segmentation. Geometric transformation. Rational polynomials. Velocity.

\section{Introdução}

O monitoramento de objetos e de pessoas é um campo de pesquisa muito explorado na área de processamento de imagens digitais (PID). Para se desenvolver um sistema de monitoramento baseado no uso de técnicas de PID, é necessário solucionar vários problemas inerentes à diversidade intrínseca aos objetos de interesse (como: cor, tamanho, forma geométrica, variabilidade nos padrões de textura e o seu próprio movimento) e problemas relacionados com as condições extrínsecas ao ambiente (como variação na intensidade da iluminação solar, chuva, sombras, interferências provocadas por outros objetos presentes na cena, etc.) [1].

Dentre os sistemas de monitoramento existentes, pode-se citar ode monitoramento do fluxo de automóveis [1], o qual apresenta um ótimo desempenho, ocasionado pela utilização da técnica de segmentação motion history image (MHI) no processo de detecção e segmentação dos corpos em movimento, tendo como ponto principal a utilização de hardware de baixo custo. O sistema de detecção e classificação de automóveis [2] utiliza a dimensão dos automóveis identificados no processo de segmentação para calcular sua altura e comprimento real, possibilitando classificá-los como carros e não carros (vans, pick-ups, caminhões).

Também é possível encontrar sistemas voltados para mensurar a velocidade de deslocamento de automóveis (corpos rígidos), como por exemplo, os sistemas desenvolvidos por [3] e [4]. O primeiro [3] mensura a velocidade de um objeto utilizando as equações de colineariedade modificadas de Ghosh [5] e os dados intrínsecos e extrínsecos do sensor de imageamento, obtidos por meio de sua calibração. Já o sistema [4] utiliza o método de calibração de câmara de Tsai em duas fases [6] e um modelo matemático para definir a relação geométrica entre o espaço-imagem e o espaço-objeto. $\mathrm{O}$ uso dos métodos de calibração dos sensores de imageamento utilizados em ambos os trabalhos [3], [4] possibilita uma diminuição dos erros causados pela distorção das lentes do sistema ótico das câmaras, porém, a utilização de ambos os sistemas torna-se inviável quando não é possível obter as informações intrínsecas dos sensores.

No caso em que se deseja mensurar a velocidade de deslocamento de um corpo por meio de imagens, há outro problema de natureza geométrica a ser resolvido, pois, devido à geometria de aquisição dos sensores de imageamento, as imagens capturadas devem ser gerenciadas pela projeção perspectiva central, fazendo todos os pontos do espaço-objeto (3D) de uma imagem serem registrados no espaço-imagem, que é $2 \mathrm{D}$, o que ocasiona perdas de dados e problemas geométricos.

O objetivo deste trabalho é apresentar um método capaz de determinara velocidade de deslocamento de corpos rígidos em tempo real por meio da análise de sequência de imagens obtidas de sensores de imageamento que não possuem suas informações intrínsecas. O sensor de imageamento deverá, antes de iniciar o procedimento de mensuração da velocidade, ser orientado, neste caso, pelo modelo de transformação baseado em funções polinomiais (MTFP). Essa orientação consiste em determinar os parâmetros de transformação do MTFP, baseado em pontos (apoio) previamente conhecidos no espaço-objeto e os seus respectivos pares no espaçoimagem. Para prover a segmentação dos corpos de interesse nas cenas, foi aplicado o método de segmentação de imagens com base na medida da diferença acumulada da média ocorrida entre os valores de pixels homólogos registrados em sucessivos quadros de imagens, desenvolvida por Bradski [7]. Ao segmentar o corpo de interesse e, aplicar o MTFP, é possível estimar as coordenadas no espaço-objeto de quaisquer pixels e quadro dessa sequência de imagens, desde que a câmara permaneça fixa em relação ao solo. Os pontos homólogos de um corpo rígido em movimento, pertencentes a dois quadros de imagens distintos, têm as suas coordenadas métricas estimadas no espaço-objeto, possibilitando, assim, a determinação da distância entre eles. Conhecendo-se o intervalo de aquisição das imagens pode-se calcular a velocidade média de deslocamento do corpo. 


\section{Segmentação de corpos em movimento pela média de Bradski}

Foi desenvolvido por Bradski e Kaehler [7], uma técnica usada para segmentar sequência de imagens em tom de cinza (nas quais, o atributo principal é o movimento de corpo) baseada nos valores médios, $\bar{M}(x, y, N)$ da equação 1, associados com cada pixel $(x, y)$ registrados em $N$ quadros consecutivos de imagem de uma mesma cena, bem como nos valores médios associados com esses mesmos pixels das diferenças acumuladas, $\bar{\Delta}(x, y, N)$ da equação 2 entre esses mesmos $N$ quadros.

$$
\begin{gathered}
\bar{M}(x, y, N)=\frac{\sum_{j=1}^{N} f\left(x, y, t_{j}\right)}{N} \\
\bar{\Delta}(x, y, N)=\frac{\sum_{j=2}^{N} f\left(x, y, t_{j}\right)-f\left(x, y, t_{j-1}\right)}{N}
\end{gathered}
$$

Tal técnica considera que os valores associados a cada pixel $(x, y)$ desses $N$ quadros consecutivos de imagens que pertençam ao intervalo $\left[L I_{x, y}, L S_{x, y}\right]$, valores de limiar inferior e superior, respectivamente, são considerados como pertencentes a elementos fixos (e, portanto, pertencentes ao fundo da imagem). Os valores $L I_{x, y}$ e $L S_{x, y}$ são determinados pelo uso da equação 3 .

$$
\begin{aligned}
& L I_{x, y}=\bar{M}(x, y, N)-\bar{\Delta}(x, y, N) k \\
& L S_{x, y}=\bar{M}(x, y, N)+\bar{\Delta}(x, y, N) k
\end{aligned}
$$

onde $k$ representa um fator de escala.

Esses dados permitem detectar pixels pertencentes aos objetos da imagem que estão se movendo entre os $\boldsymbol{N}$ quadros de imagens considerados e, podem ser segmentados por valores de limiar calculados pela equação 3 .

\section{Modelo polinomial 3D}

É possível relacionar o espaço-imagem e o espaço-objeto por meio do modelo polinomial 3D. A forma resumida de um polinômio simples $3 \mathrm{D}$ pode ser dada pelas equações 4 e 5 :

$$
\begin{aligned}
& l=\sum_{i=0}^{m} \sum_{j=0}^{n} \sum_{k=0}^{p} a_{i j k} x^{i} y^{j} z^{k} \\
& c=\sum_{i=0}^{m} \sum_{j=0}^{n} \sum_{k=0}^{p} b_{i j k} x^{i} y^{j} z^{k}
\end{aligned}
$$

onde $\boldsymbol{c}$ e $\boldsymbol{l}$ representam as coordenadas coluna e linha de um determinado pixel na imagem; $x, y$ e $z$ são as coordenadas cartesianas tridimensionais do ponto no terreno; $a_{i j k}$, e $b_{i j k}$ são os coeficientes polinomiais; $m, n$ e $p$ são valores inteiros, pertencentes ao intervalo $[0,3]$, com $m+n p$ sendo a ordem das funções polinomiais, geralmente três.

As equações 6 e 7 mostram com mais detalhes um exemplo de expansão das notações referentes às equações 4 e 5, no caso, um polinômio de terceira ordem no espaço 3D.

$$
\begin{aligned}
l=a_{000}+a_{010} y & +a_{100} x+a_{001} z+a_{110} y x+a_{011} y z+a_{101} x z+a_{020} y^{2}+a_{200} x^{2}+a_{002} z^{2} \\
& +a_{111} x y z+a_{030} y^{3}+a_{210} y x^{2}+a_{012} y z^{2}+a_{120} y^{2} x+a_{300} x^{3}+a_{102} x z^{2} \\
& +a_{021} y^{2} z+a_{201} x^{2} z+a_{003} z^{3}
\end{aligned}
$$




$$
\begin{aligned}
c=b_{000}+b_{010} y & +b_{100} x+b_{001} z+b_{110} y x+b_{011} y z+b_{101} x z+b_{020} y^{2}+b_{200} x^{2}+b_{002} z^{2} \\
& +b_{111} x y z+b_{030} y^{3}+b_{210} y x^{2}+b_{012} y z^{2}+b_{120} y^{2} x+b_{300} x^{3}+b_{102} x z^{2} \\
& +b_{021} y^{2} z+b_{201} x^{2} z+b_{003} z^{3}
\end{aligned}
$$

Existem padrões de distorções ou de deslocamentos que podem ser modelados ou corrigidos por termos específicos do polinômio (Figura 1). A principal vantagem do uso do modelo polinomial é a correção de todas as fontes de distorção simultaneamente [9].

Figura 1: Padrões de distorção modelados por termos específicos de um polinômio [8].

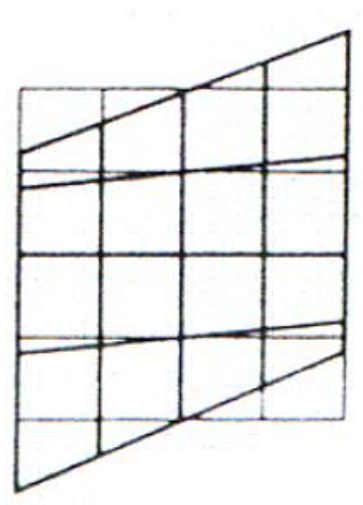

$$
a_{7} x y^{2}
$$

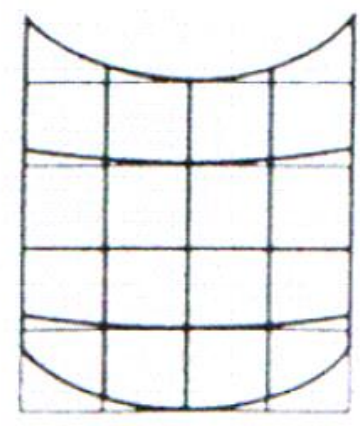

$a_{14} x^{2} y^{2}$

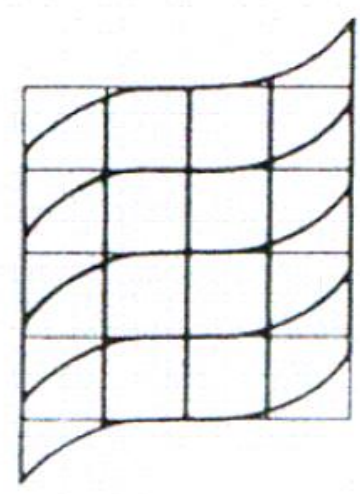

$a_{8} x^{3}$

\section{Sistema desenvolvido}

$\mathrm{Na}$ evolução deste trabalho, foi desenvolvido um sistema (protótipo) computacional capaz de mensurar a velocidade de deslocamento de um corpo rígido em movimento. Esse sistema é composto por cinco módulos: 1) o módulo de interface, responsável por realizar a interface entre o sistema computacional e a câmara, capturando as imagens adquiridas pela câmara e disponibilizando-as para processamento; 2) o módulo responsável por realizar a detecção dos corpos em movimento em uma sequência de imagens adquiridas, utilizando o método de segmentação baseado na média de Bradski; 3) o módulo de conversão de coordenadas, responsável por realizar a conversão de coordenadas do espaço-imagem para o espaço-objeto por meio do modelo polinomial, o qual tem como parâmetro de entrada o conhecimento, a priori, de 21 pontos do espaço-objeto com os respectivos pontos correspondentes no espaço-imagem; 4) o módulo responsável por mensurar a velocidade média de deslocamento de um corpo e proceder com as análises relativas a esse valor; e 5) o módulo responsável por exibir as sequências de imagens com sobreposição dos detalhes computados. Essa implementação está representada no fluxograma da Figura 2. 
Figura 2: Fluxograma do sistema.

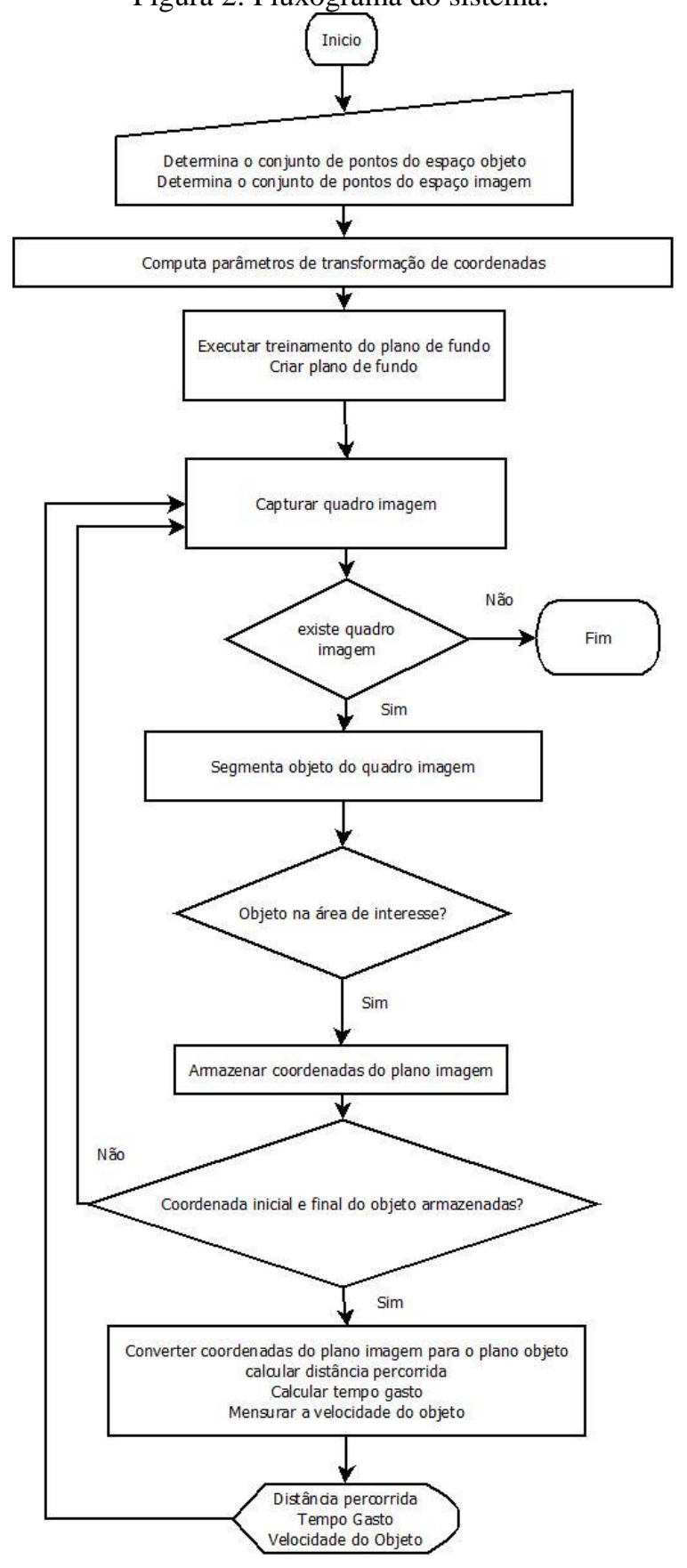

\subsection{Experimento realizado}

Para validar o sistema e a metodologia desenvolvida, foi realizado um experimento, em um laboratório de Física aplicada, que contou com a utilização de um aparelho denominado trilho de ar para mensurar a velocidade de deslocamento de um corpo sobre uma superfície. Esse aparelho possui orifícios laterais, no qual, ar comprimido é injetado pelo seu interior e, ao sair pelos orifícios laterais, forma um colchão de ar na superfície, permitindo que um corpo deslize sem que haja, ou que seja insignificante, a atuação da força de atrito entre as superfícies do corpo e do trilho.

Cinco sensores fotoelétricos são colocados sobre esse trilho. Cada sensor está conectado a um cronômetro (com precisão de $5 \times 10^{-4}$ segundos). As distâncias entre esses sensores são conhecidas. No momento em que o 
corpo se desloca pelo trilho, passando pelo primeiro sensor, todos os cronômetros são acionados, a partir do zero. Quando o corpo passa por outro sensor, o cronômetro ligado a esse sensor é paralisado. Com isso, tem-se a distância percorrida pelo corpo entre cada sensor e quanto tempo foi necessário para percorrer cada uma dessas distâncias. Com esses dados, é possível calcular a velocidade, assumida como real, do corpo em questão em todos os pontos cronometrados.

O experimento foi registrado em sequência de imagens, sendo submetidas ao módulo de processamento de imagens do sistema desenvolvido, o qual utilizou a técnica de detecção de movimento e segmentação de sequência de imagens em tempo real desenvolvido por Bradski e Kaehler [7] para identificar o corpo em movimento. O sistema implementado captura, automaticamente, dois quadros consecutivos de imagens, nos instantes $t_{i}$ e $t_{i+1}$, que são analisados para detectar se um corpo qualquer alterou a sua posição. Se houve alguma alteração na posição do corpo, é delimitado um retângulo envolvente, cujas coordenadas do canto inferior esquerdo são utilizadas nos cálculos posteriores (Figura 3).

Figura 3: Sequência de imagens. (a) Quadro $i$. (b) Quadro $i+1$. (c) Movimento detectado (o vetor $\boldsymbol{d}$ aponta para a borda esquerda inferior da imagem) e objeto em movimento é envolto pelo quadrilátero mínimo (em branco).

(d) Extração da coordenada do ponto (em amarelo) do lado esquerdo da borda inferior do quadrilátero.

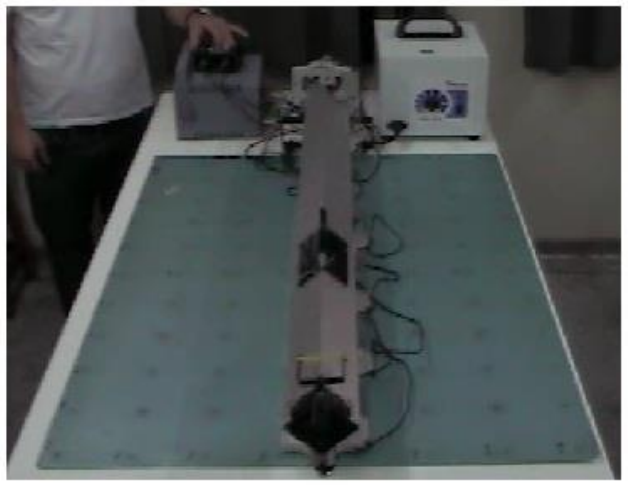

(a)

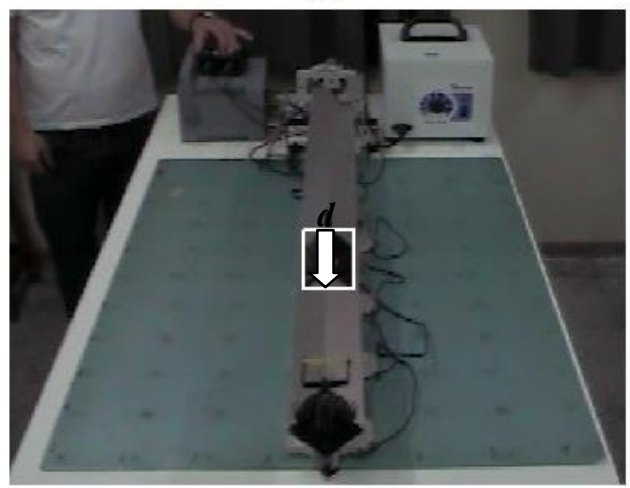

(c)

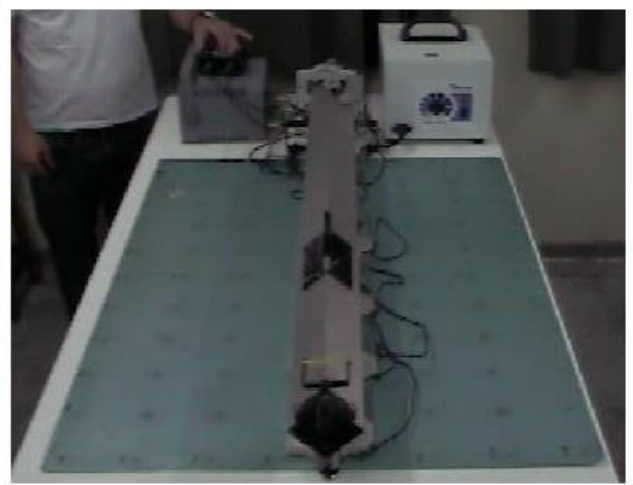

(b)

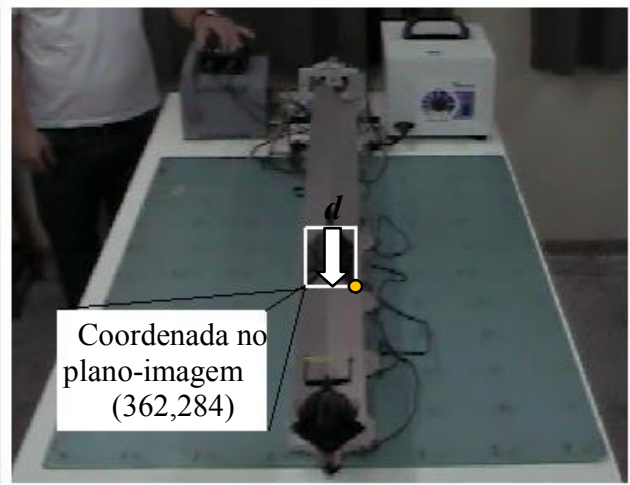

(d)

Para determinara velocidade do objeto, é necessário determinar dois parâmetros: 1) o intervalo de tempo em que esse deslocamento foi observado $(\Delta t)$; e 2) o espaço percorrido $(\Delta s)$, em unidades métricas, pelo corpo durante essa observação.

O intervalo de tempo da observação é obtido por meio da quantidade de quadros que a câmara consegue imagear por segundo. Para cada quadro adquirido $i$, o momento $t_{i}$ em que isso ocorreu também é registrado, logo, para dois quadros consecutivos de imagens, tem-se:

$$
\Delta t=t_{i+1}-t_{i}
$$


A determinação do espaço percorrido $(\Delta s)$ deve ser realizada no espaço-objeto (3D - mundo real), para tanto, o ponto medido no espaço-imagem (2D) deve ser transformado (equações 6 e 7) para que a determinação da velocidade seja efetivamente calculada.

A fim de que essa transformação seja utilizada na determinação das coordenadas cartesianas 3D (espaço-objeto) os parâmetros $a_{i j k}$ e $b_{i j k}$ (no total de 40) devem ser determinados, sendo necessários pelo menos 21 pontos de apoio (coordenadas 3D conhecidas no espaço-objeto) perfeitamente foto identificados, referenciando as duas imagens ao sistema de coordenadas do espaço-objeto. Dessa forma, qualquer ponto identificado em qualquer uma das imagens pode ter as suas coordenadas 3D definidas, possibilitando a determinação do espaço percorrido e, consequentemente, o cálculo da velocidade do objeto, considerando que o mesmo objeto tenha se deslocado e suas coordenadas tenham sido capturadas nas duas imagens.

$\mathrm{Na}$ realização do experimento em laboratório, foi utilizada uma placa de aço contendo a marcação de 88 pontos visíveis, distribuídos em um arranjo matricial, regularmente espaçado de $100 \mathrm{~mm} \mathrm{em} 100 \mathrm{~mm}$. Essa placa (Figura 4) foi usada como elemento de fundo da cena para aquisição das imagens para estabelecer um sistema de coordenadas cartesianas 3D, atribuindo coordenadas $\mathrm{X}, \mathrm{Y}$ e $\mathrm{Z}$ aos pontos dessa placa. Ao adquirir as imagens, alguns pontos são escolhidos como de apoio, que devem ser medidos no espaço-imagem, possibilitando a determinação dos parâmetros de transformação das equações 6 e 7.

Figura 4: Sistema do trilho de ar usado para calcular a velocidade de deslocamento de um corpo rígido em condições controladas.

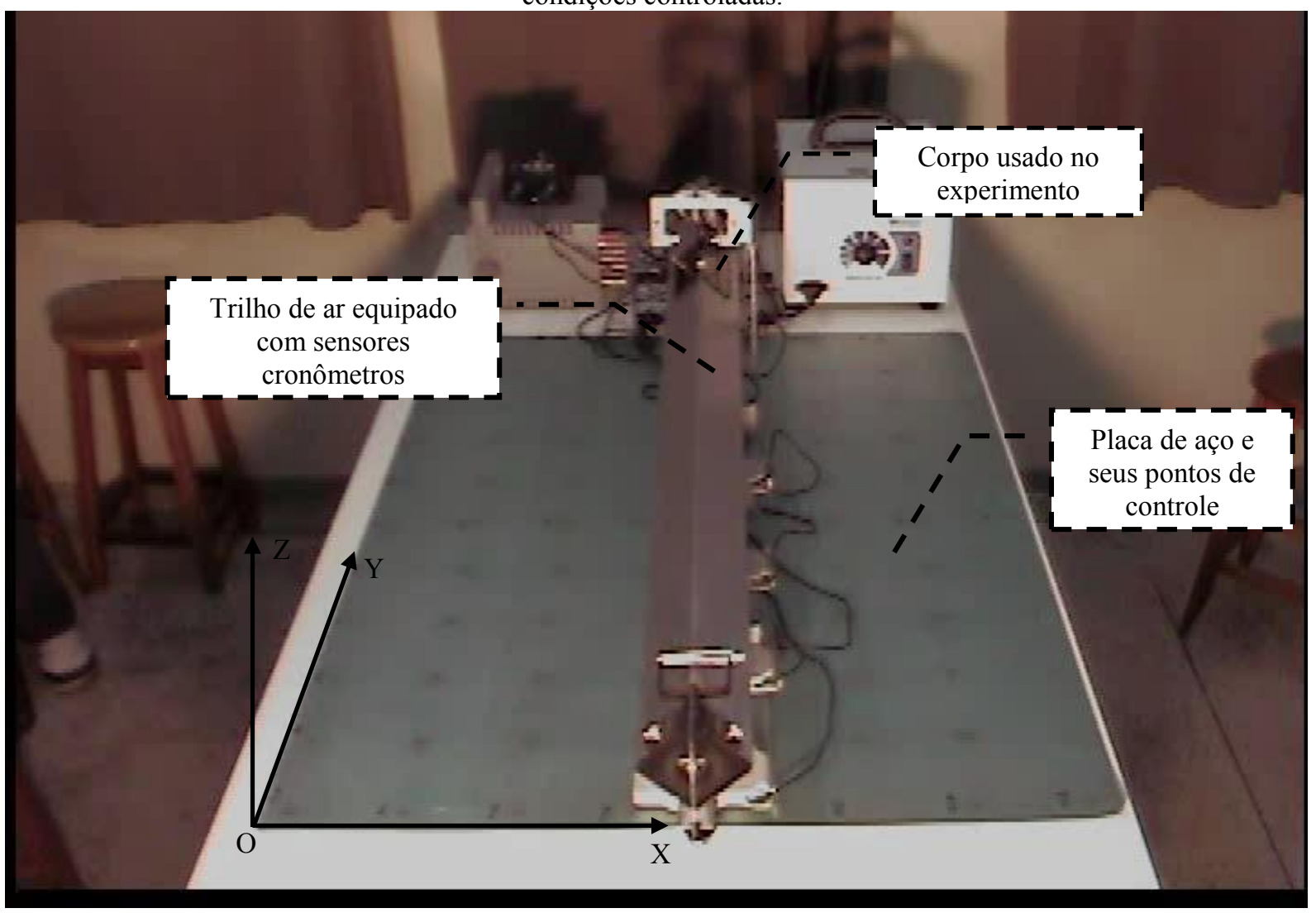

Conhecido os parâmetros de transformação, qualquer ponto na imagem pode ter sua coordenada no espaço-objeto estimada com relativa precisão. Aplicando a equação da distância euclidiana nas coordenadas 3D no espaço-objeto nos dois instantes determina-se o espaço percorrido $(\Delta s)$, o qual é utilizado na determinação da velocidade do corpo. 


\subsection{Procedimentos para a condução do experimento}

A Figura 5 ilustra o ambiente montado para a execução do experimento. Sob o trilho de ar, foi instalada a placa de aço (Figura 4), que permite determinar pontos que relacionam o espaço-objeto com o plano-imagem. O experimento do corpo deslocando-se sobre o trilho de ar foi filmado e as imagens adquiridas foram submetidas ao sistema proposto e, por meio dessas, calculou-se a velocidade de deslocamento do corpo (velocidade calculada por análise de imagens e cálculos fotogramétricos). Esse valor da velocidade é comparado com o valor da velocidade real, computada pelos sensores do trilho de ar.

Figura 5: Ambiente montado para o experimento.

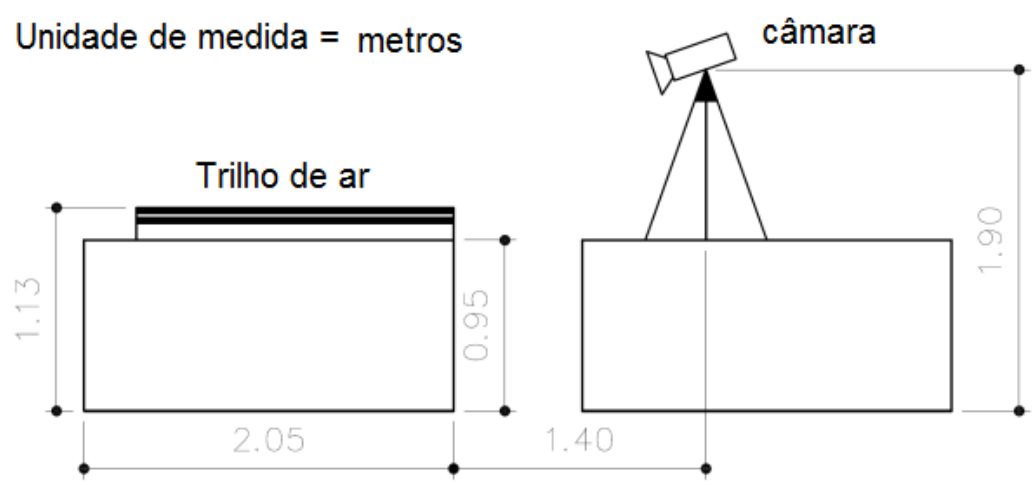

\subsection{Resultados obtidos}

Esta sessão descreve os resultados referentes ao cálculo da velocidade de deslocamento em linha reta de um corpo rígido com aceleração nula. Para isso, realizou-se uma regressão linear, utilizando o método dos mínimos quadrados (MMQ) para possibilitar a interpolação da velocidade, feito por meio do modelo linear $y=b+a x$.

\subsubsection{Resultado computado com a utilização de sensores fotoelétricos}

A Tabela 1 exibe a velocidade de deslocamento de um corpo rígido, computado por sensores presentes no trilho de ar.

Tabela 1: Dados obtidos nos experimentos em laboratório e cálculo das respectivas velocidades e desvios

\begin{tabular}{|c|c|c|c|c|c|c|}
\hline & \multicolumn{5}{|c|}{ Dados experimentais } & \multirow{3}{*}{$\begin{array}{c}\text { Velocidade } \\
\text { calculada } \\
(\mathrm{cm} / \mathrm{s})\end{array}$} \\
\hline & \multicolumn{5}{|c|}{ intervalos de tempos obtidos em cada experimento } & \\
\hline & $\begin{array}{l}t_{0} \\
(\mathrm{~s})\end{array}$ & $\begin{array}{l}\Delta t_{1} \\
\text { (s) }\end{array}$ & $\begin{array}{l}\Delta t_{2} \\
(\mathrm{~s})\end{array}$ & $\begin{array}{l}\Delta t_{3} \\
(\mathrm{~s})\end{array}$ & $\begin{array}{l}\Delta t_{4} \\
(\mathrm{~s})\end{array}$ & \\
\hline Experimento 1 & 0,0000 & 0,5150 & 1,0620 & 1,5540 & 2,1230 & $28,4 \pm 0,3$ \\
\hline Experimento 2 & 0,0000 & 0,5200 & 1,0730 & 1,5690 & 2,1470 & $28,1 \pm 0,3$ \\
\hline Experimento 3 & 0,0000 & 0,5200 & 1,0780 & 1,6180 & 2,2710 & $26,6 \pm 0,6$ \\
\hline Experimento 4 & 0,0000 & 0,5210 & 1,0760 & 1,5780 & 2,1610 & $27,9 \pm 0,3$ \\
\hline$\overline{\Delta t}(\mathrm{~s})$ & 0,0000 & 0,5190 & 1,0722 & 1,5798 & 2,1755 & $27,7 \pm 0,4$ \\
\hline$\Delta s(\mathrm{~cm})$ & 0,0 & 15,0 & 30,0 & 45,0 & 60,0 & \\
\hline
\end{tabular}

$\overline{\Delta t}$ - Valor médio dos intervalos de tempo.

$\Delta t_{i}$ - Valor de cada intervalo de tempo medidos nos experimentos.

$\Delta s-$ Valor do espaço percorrido em cada intervalo de tempo.

$t_{0}$ - Instante inicial (ajustado para zero segundos). 
Os dados de tempo coletados nos quatro experimentos foram usados no cômputo dos respectivos valores médios $\overline{\Delta t}$. Esses valores médios minimizam a ocorrência de erros sistemáticos ocorridos no desenvolvimento desses experimentos. Somente esses valores médios são usados nas análises feitas neste trabalho.

Figura 6: Ajuste linear das distâncias percorridas $\Delta s$ pelo MMQ em função dos intervalos de tempo médios $\overline{\Delta t}$.

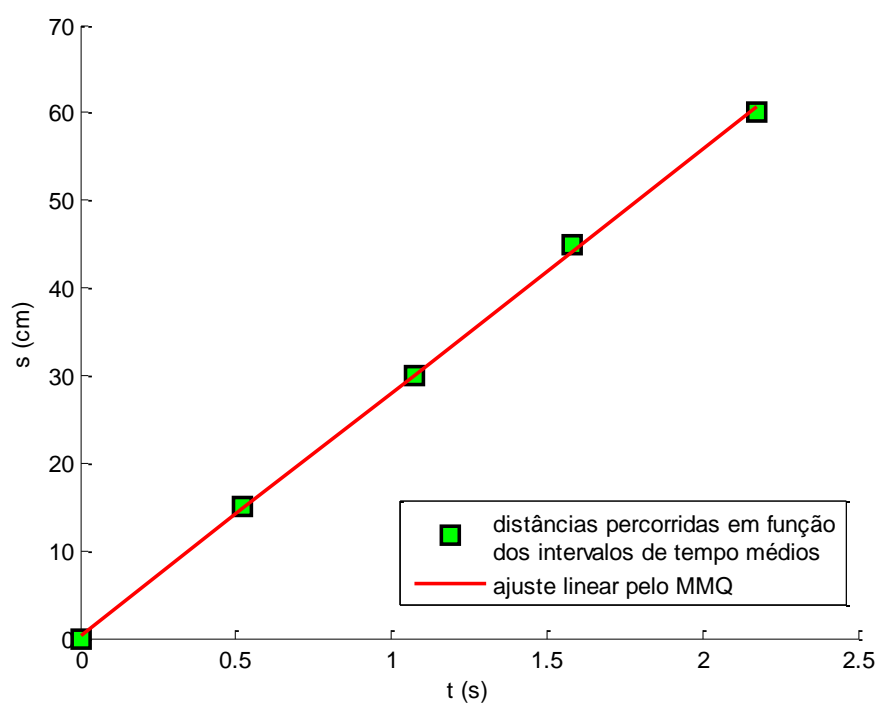

- Equação da reta (Figura 6) calculada pelo método dos mínimos quadrados (MMQ): $s=0,4+27,7 t$.

- Fator de correlação da reta calculada pelo MMQ: 0,9997. Esse valor mostra que os dados experimentais estão fortemente correlacionados com o modelo linear do movimento retilíneo uniforme $s=s_{0}+v t$, onde: $s$ é o espaço percorrido a partir de uma posição inicial $s_{0}$ em função do tempo $t$ com velocidade constante $v$.

\subsubsection{Resultado computado por meio do método de transformação polinomial utilizando segmentação automática de objetos}

A Tabela 2 ilustra os dados obtidos por meio do software implementado, utilizando o método de segmentação desenvolvido [7], no processo de segmentação, e o método de conversão de coordenadas do planoimagem para o espaço-objeto baseado no método de transformação polinomial.

Esses valores de distância percorrida e de intervalo de tempo decorrido foram extraídos das sequências de quadros de imagens, os quais obtiveram uma velocidade média de 33,484 centímetros por segundo com um fator de correlação equivalente a 0,999 (Figura 7).

Comparando os valores da velocidade média obtida por meio do método baseado no método de transformação polinomial $(33,484 \mathrm{~cm} / \mathrm{s})$, com o valor obtido por sensores fotoelétricos $(27,7 \mathrm{~cm} / \mathrm{s})$, evidencia-se que o desvio cometido é da ordem de $20,88 \%$. 
Tabela 2: Dados obtidos nos experimentos em laboratório utilizando o método de conversão de coordenadas do plano-imagem para o espaço-objeto baseado no método de transformação polinomial

\begin{tabular}{|c|c|c|c|c|c|c|c|}
\hline \multicolumn{8}{|c|}{ Captura automática } \\
\hline \multirow{2}{*}{$\begin{array}{l}\text { Número do } \\
\text { quadro }\end{array}$} & \multicolumn{2}{|c|}{$\begin{array}{l}\text { Coordenadas no } \\
\text { plano-imagem }\end{array}$} & \multicolumn{2}{|c|}{$\begin{array}{l}\text { Coordenadas no } \\
\text { espaço-objeto }\end{array}$} & \multirow{2}{*}{$\begin{array}{l}\text { Intervalo } \\
\text { de tempo } \\
\text { transcorrido } \\
(\text { seg })\end{array}$} & \multicolumn{2}{|c|}{ Distância mensurada } \\
\hline & $\mathrm{Xi}$ & $\mathrm{Yi}$ & Xo & Yo & & $\mathrm{mm}$ & $\mathrm{cm}$ \\
\hline 1 & 367 & 211 & 311,122 & 803,369 & 0,000000 & 0,00000 & 0,00000 \\
\hline 2 & 367 & 213 & 311,428 & 791,296 & 0,068966 & 12,0729 & 1,20729 \\
\hline 3 & 367 & 216 & 311,868 & 773,609 & 0,103448 & 29,7602 & 2,97602 \\
\hline 4 & 367 & 218 & 312,150 & 762,081 & 0,137931 & 41,2881 & 4,12881 \\
\hline 5 & 366 & 218 & 309,811 & 762,061 & 0,172414 & 41,3081 & 4,13081 \\
\hline 7 & 367 & 223 & 312,819 & 734,107 & 0,241379 & 69,2619 & 6,92619 \\
\hline 8 & 367 & 226 & 313,198 & 717,857 & 0,275862 & 85,5121 & 8,55121 \\
\hline 9 & 367 & 228 & 313,442 & 707,229 & 0,310345 & 96,1401 & 9,61401 \\
\hline 10 & 367 & 231 & 313,797 & 691,577 & 0,344828 & 111,792 & 11,1792 \\
\hline 11 & 367 & 233 & 314,026 & 681,327 & 0,379310 & 122,042 & 12,2042 \\
\hline 12 & 366 & 235 & 312,035 & 671,205 & 0,413793 & 132,164 & 13,2164 \\
\hline 13 & 366 & 237 & 312,265 & 661,230 & 0,448276 & 142,139 & 14,2139 \\
\hline 14 & 367 & 240 & 314,786 & 646,518 & 0,482759 & 156,851 & 15,6851 \\
\hline 15 & 368 & 242 & 317,165 & 636,864 & 0,517241 & 166,505 & 16,6505 \\
\hline 16 & 368 & 245 & 317,448 & 622,590 & 0,551724 & 180,779 & 18,0779 \\
\hline 18 & 369 & 250 & 320,027 & 599,356 & 0,620690 & 204,013 & 20,4013 \\
\hline 19 & 369 & 253 & 320,271 & 585,722 & 0,655172 & 217,647 & 21,7647 \\
\hline 20 & 369 & 255 & 320,430 & 576,755 & 0,689655 & 226,614 & 22,6614 \\
\hline 21 & 369 & 257 & 320,586 & 567,883 & 0,724138 & 235,486 & 23,5486 \\
\hline 22 & 370 & 260 & 322,891 & 554,746 & 0,758621 & 248,623 & 24,8623 \\
\hline 23 & 370 & 263 & 323,098 & 541,803 & 0,793103 & 261,566 & 26,1566 \\
\hline 24 & 370 & 265 & 323,234 & 533,280 & 0,827586 & 270,090 & 27,0090 \\
\hline 25 & 378 & 267 & 339,721 & 524,810 & 0,862069 & 278,559 & 27,8559 \\
\hline 26 & 379 & 270 & 341,839 & 512,283 & 0,896552 & 291,086 & 29,1086 \\
\hline 27 & 378 & 273 & 339,891 & 499,941 & 0,931034 & 303,428 & 30,3428 \\
\hline 28 & 379 & 276 & 341,979 & 487,747 & 0,965517 & 315,622 & 31,5622 \\
\hline 29 & 378 & 279 & 340,054 & 475,726 & 1,000000 & 327,643 & 32,7643 \\
\hline 30 & 376 & 282 & 336,172 & 463,863 & 1,034480 & 339,506 & 33,9506 \\
\hline
\end{tabular}


Figura 7: Ajuste linear das distâncias percorridas $\Delta s$ pelo MMQ em função dos intervalos de tempo $\Delta t$ decorridos.

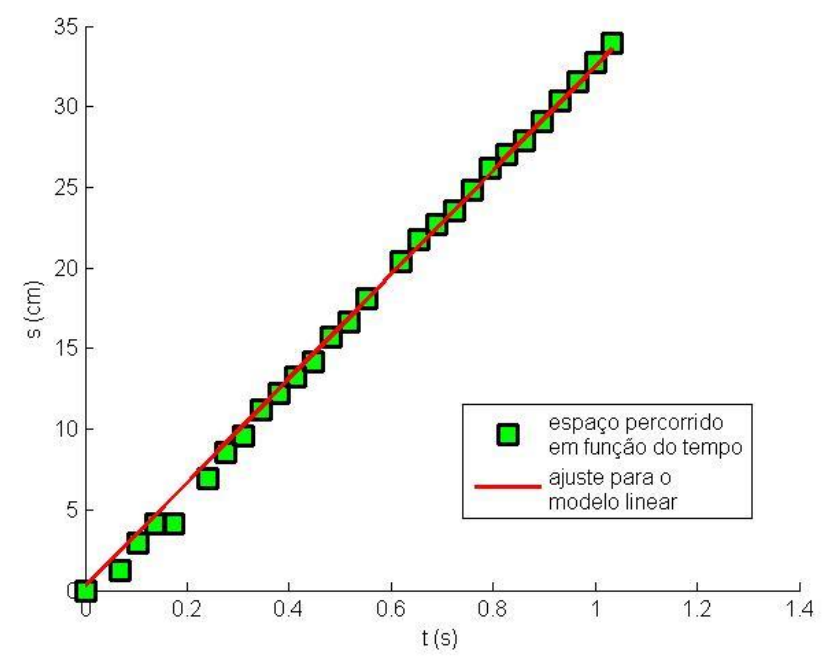

\subsubsection{Resultado computado por meio do método de transformação polinomial utilizando segmentação manual de objetos}

A Tabela 3 ilustra os dados obtidos pela captura manual dos pontos no plano-imagem, utilizando o método de conversão de coordenadas do plano-imagem para o espaço-objeto, utilizando o método de transformação polinomial.

Esses valores de distância percorrida e de intervalo de tempo decorrido foram extraídos das sequências de quadros de imagens, os quais obtiveram uma velocidade média de 34,99 centímetros por segundo com um fator de correlação equivalente a 0,9983 (Figura 8).

Figura 8: Ajuste linear das distâncias percorridas $\Delta s$ pelo MMQ em função dos intervalos de tempo $\Delta t$ decorridos.

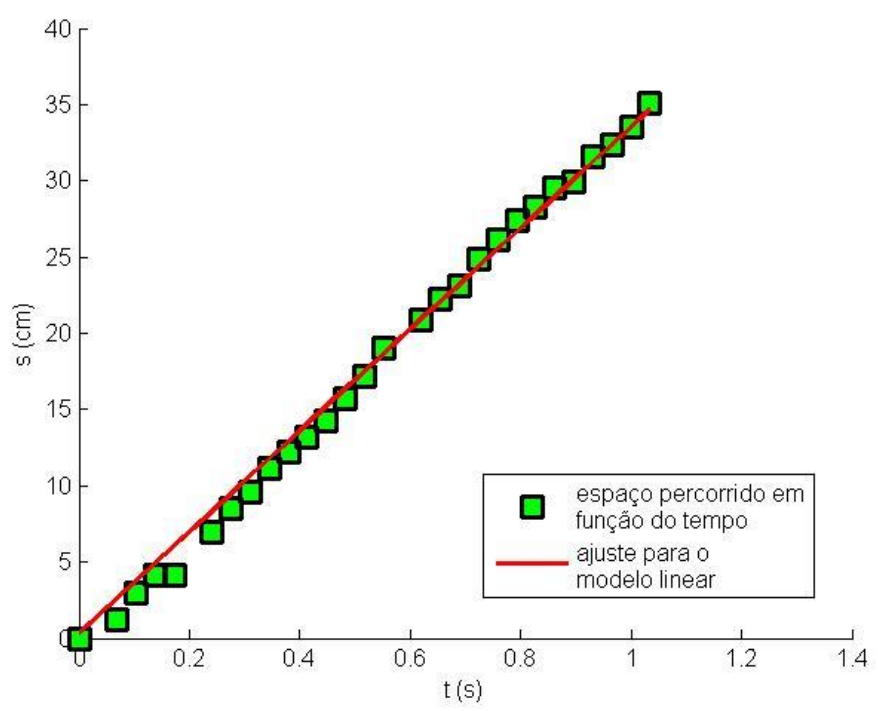


Comparando os valores da velocidade média obtida por meio do método baseado no método de transformação polinomial $(34,99 \mathrm{~cm} / \mathrm{s})$, com o valor obtido por sensores fotoelétricos $(27,70 \mathrm{~cm} / \mathrm{s})$, conclui-se que o desvio cometido é da ordem de $26,31 \%$.

Tabela 3: Dados obtidos nos experimentos em laboratório utilizando o método de conversão de coordenadas do plano-imagem para o espaço-objeto utilizando o método de transformação polinomial 2D 3D

\begin{tabular}{|c|c|c|c|c|c|c|c|}
\hline \multicolumn{8}{|c|}{ Captura Manual } \\
\hline \multirow{2}{*}{$\begin{array}{l}\text { Número do } \\
\text { quadro }\end{array}$} & \multicolumn{2}{|c|}{$\begin{array}{l}\text { Coordenadas no } \\
\text { plano-imagem }\end{array}$} & \multicolumn{2}{|c|}{$\begin{array}{l}\text { Coordenadas no } \\
\text { espaço-objeto }\end{array}$} & \multirow{2}{*}{$\begin{array}{l}\text { Intervalo de } \\
\text { tempo } \\
\text { transcorrido } \\
(\text { seg })\end{array}$} & \multicolumn{2}{|c|}{ Distância mensurada } \\
\hline & $\mathrm{Xi}$ & $\mathrm{Yi}$ & Xo & Yo & & $\mathrm{mm}$ & $\mathrm{cm}$ \\
\hline 1 & 367 & 211 & 311,122 & 803,369 & 0,000000 & 0,00000 & 0,00000 \\
\hline 2 & 367 & 213 & 311,428 & 791,296 & 0,068966 & 12,0729 & 1,20729 \\
\hline 3 & 367 & 216 & 311,868 & 773,609 & 0,103448 & 29,7602 & 2,97602 \\
\hline 4 & 367 & 218 & 312,150 & 762,081 & 0,137931 & 41,2881 & 4,12881 \\
\hline 5 & 366 & 218 & 309,811 & 762,061 & 0,172414 & 41,3081 & 4,13081 \\
\hline 7 & 367 & 223 & 312,819 & 734,107 & 0,241379 & 69,2619 & 6,92619 \\
\hline 8 & 367 & 226 & 313,198 & 717,857 & 0,275862 & 85,5121 & 8,55121 \\
\hline 9 & 367 & 228 & 313,442 & 707,229 & 0,310345 & 96,1401 & 9,61401 \\
\hline 10 & 367 & 231 & 313,797 & 691,577 & 0,344828 & 111,792 & 11,1792 \\
\hline 11 & 367 & 233 & 314,026 & 681,327 & 0,379310 & 122,042 & 12,2042 \\
\hline 12 & 366 & 235 & 312,035 & 671,205 & 0,413793 & 132,164 & 13,2164 \\
\hline 13 & 366 & 237 & 312,265 & 661,230 & 0,448276 & 142,139 & 14,2139 \\
\hline 14 & 365 & 240 & 310,418 & 646,498 & 0,482759 & 156,871 & 15,6871 \\
\hline 15 & 365 & 243 & 310,761 & 632,052 & 0,517241 & 171,317 & 17,1317 \\
\hline 16 & 366 & 247 & 313,344 & 613,201 & 0,551724 & 190,168 & 19,0168 \\
\hline 18 & 364 & 251 & 309,502 & 594,756 & 0,620690 & 208,613 & 20,8613 \\
\hline 19 & 364 & 254 & 309,823 & 581,200 & 0,655172 & 222,169 & 22,2169 \\
\hline 20 & 365 & 256 & 312,126 & 572,290 & 0,689655 & 231,079 & 23,1079 \\
\hline 21 & 364 & 260 & 310,437 & 554,725 & 0,724138 & 248,644 & 24,8644 \\
\hline 22 & 364 & 263 & 310,732 & 541,787 & 0,758621 & 261,583 & 26,1583 \\
\hline 23 & 364 & 266 & 311,018 & 529,036 & 0,793103 & 274,334 & 27,4334 \\
\hline 24 & 364 & 268 & 311,205 & 520,635 & 0,827586 & 282,734 & 28,2734 \\
\hline 25 & 364 & 271 & 311,479 & 508,180 & 0,862069 & 295,190 & 29,5190 \\
\hline 26 & 364 & 272 & 311,569 & 504,065 & 0,896552 & 299,304 & 29,9304 \\
\hline 27 & 364 & 276 & 311,920 & 487,790 & 0,931034 & 315,579 & 31,5579 \\
\hline 28 & 364 & 278 & 312,092 & 479,757 & 0,965517 & 323,612 & 32,3612 \\
\hline 29 & 364 & 281 & 312,344 & 467,836 & 1,000000 & 335,533 & 33,5533 \\
\hline 30 & 364 & 285 & 312,671 & 452,169 & 1,034480 & 351,201 & 35,1201 \\
\hline
\end{tabular}




\subsubsection{Análise dos resultados}

A Tabela 4 ilustra os resultados obtidos com os testes realizados, destacando as velocidades mensuradas por meio da detecção automática de corpos rígidos, utilizando o método de segmentação de descrito nos trabalhos de Bradski e Kaehler [7] e por meio da detecção manual. Destacam-se, também, o valor percentual do erro ocasionado pela identificação automática dos corpos rígidos no processo de mensuração de velocidade e o percentual de erro dos métodos de conversão de coordenadas do espaço-imagem para o espaço-objeto em comparação com a velocidade obtida por meio dos sensores fotoelétricos.

Tabela 4: Resultados dos testes realizados no processo de mensuração da velocidade média

\begin{tabular}{|c|c|c|c|c|c|}
\hline \multirow[t]{2}{*}{$\begin{array}{l}\text { Método utilizado no } \\
\text { processo de mensuração da } \\
\text { velocidade }\end{array}$} & \multicolumn{2}{|c|}{$\begin{array}{l}\text { Velocidades }(\mathrm{cm} / \mathrm{s}) \text { obtidas } \\
\text { com a identificação de objetos }\end{array}$} & \multirow{2}{*}{$\begin{array}{c}\text { Erro entre } \\
\text { a captura de } \\
\text { pontos } \\
\text { manual e } \\
\text { automática \% }\end{array}$} & \multicolumn{2}{|c|}{$\begin{array}{c}\text { Erro com relação aos } \\
\text { sensores fotoelétricos \% } \\
\text { (velocidade medida por meio } \\
\text { da identificação de objetos) }\end{array}$} \\
\hline & Automática & Manual & & Automática & Manual \\
\hline Sensores fotoelétricos & 27,70 & ------ & 0,00 & 00,00 & 00,00 \\
\hline $\begin{array}{l}\text { Método de transformação } \\
\text { polinomial }\end{array}$ & 33,48 & 34,99 & 4,30 & 20,80 & 26,31 \\
\hline
\end{tabular}

Com base nos resultados obtidos, pode-se dizer que a técnica utilizada para a identificação automática dos corpos rígidos em movimento ocasiona um erro médio de $4,30 \%$ no processo de mensuração da velocidade média, isso se deve ao fato de identificar erroneamente o canto inferior esquerdo (Figura 9), utilizado como ponto de referência para realizar a mensuração da velocidade média.

Figura 9: Captura de um corpo rígido em movimento com ponto de referência identificado erroneamente.

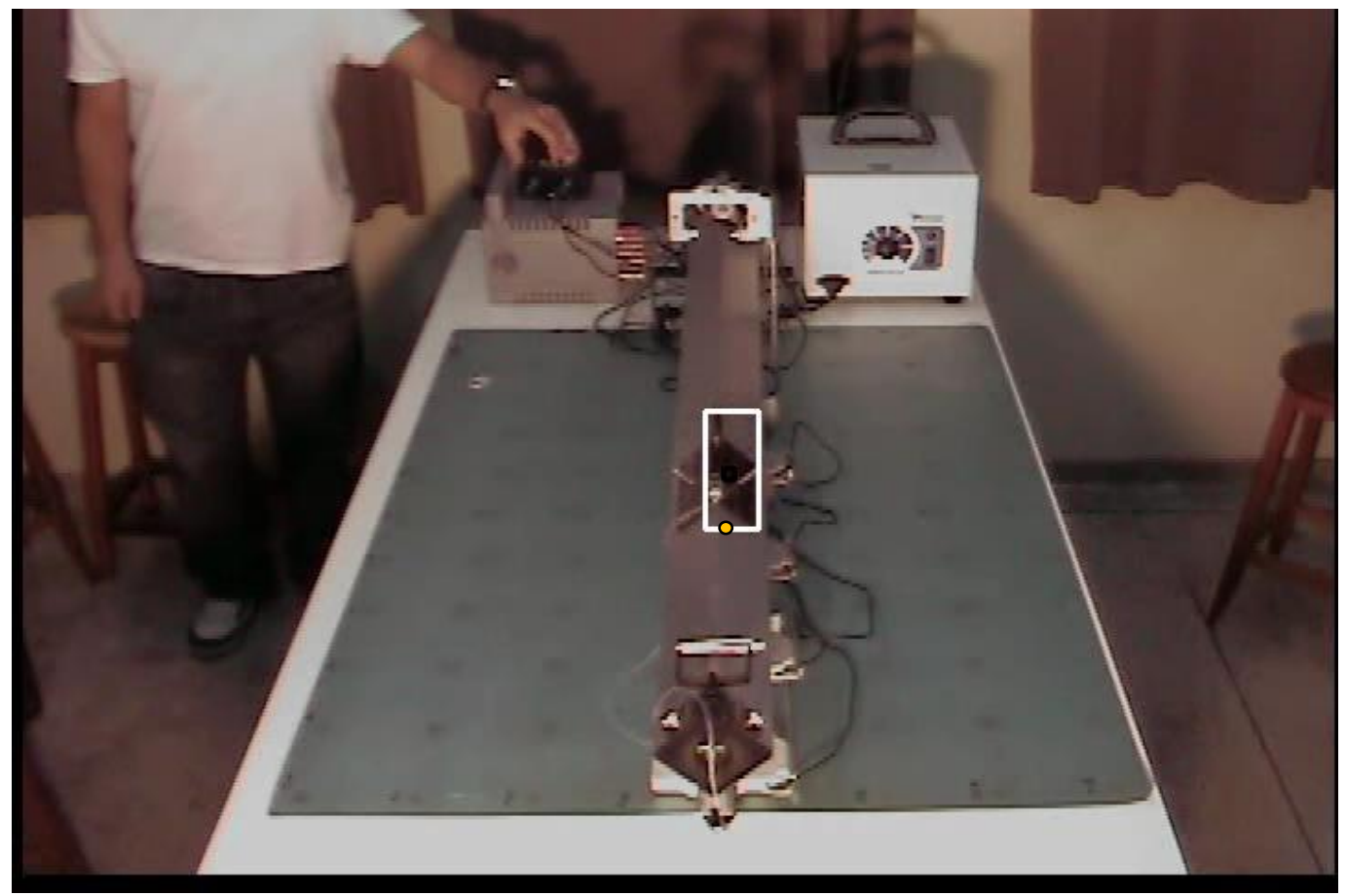




\section{Considerações finais}

Este trabalho apresentou uma metodologia para a mensuração da velocidade de um corpo rígido em movimento, tendo, como ponto de partida, uma sequência de imagens obtidas por uma filmadora digital. Foi implementado um protótipo para adquirir, selecionar, detectar e mensurar a velocidade de um corpo de forma automática, utilizando a transformação polinomial, cujos resultados foram apresentados nos experimentos.

Pelos experimentos, verifica-se que foi estimada a velocidade de $33,48 \mathrm{~cm} / \mathrm{s}$, com erro de $20,8 \%$, em relação à velocidade obtida $(27,70 \mathrm{~cm} / \mathrm{s})$ por meio dos sensores fotoelétricos. Já utilizando a detecção de corpos, de maneira manual, a aplicação do método de transformação polinomial apresentou nos experimentos uma velocidade estimada de $34,99 \mathrm{~cm} / \mathrm{s}$, com erro de $26,32 \%$ em relação à velocidade obtida pelos sensores fotoelétricos. Os problemas apresentados pelo processo de segmentação das imagens mostram a necessidade de uma investigação maior, inclusive com a avaliação de outras técnicas de segmentação. A variação da escala e a determinação de um ponto na imagem correspondente a uma parte do corpo com pouca elevação foram identificadas como fatores que, com um estudo mais pormenorizado, podem trazer melhores resultados. Outro ponto que também necessita maior investigação é o erro ocasionado pela variação da taxa de captura das imagens por meio da filmadora, a qual varia entre 30 e 29 quadros por segundo.

O protótipo implementado e testado em condições controladas, com as devidas considerações, demonstra que a metodologia é válida, porém, recomenda-se a realização de outros testes experimentais, principalmente, com o uso de diferentes ângulos de visada da câmara e diferentes distâncias entre a câmara e a cena (fator de escala), para saber como esses fatores influenciam no resultado.

Pretende-se utilizar, em trabalhos futuros, outras técnicas de segmentação e de conversão de coordenadas entre o espaço-imagem e espaço-objeto, visando a melhorar a mensuração da velocidade de deslocamento dos corpos rígidos.

\section{Referências}

[1] MONTANHA, A. Especificação de um sistema para monitoração de fluxo de veículos. Dissertação (Mestrado em Ciência da Computação) Universidade Estadual de Maringá (UEM), Maringa, 2010.

[2] GUPTE, S.et al. Detection and classification of vehicles, IEEE Transactions on Intelligent Transportation Systems, v. 3, n. 1, March 2002.

[3] KOYAMA, C, S. Determinação da velocidade de um objeto, a partir de uma sequência de imagens, aplicando as equações de colineariedade modificadas. Dissertação (Mestrado em Ciências Cartográficas), Universidade Estadual Paulista(UNESP), Presidente Prudente, 2001.

[4] YAN YAN, Shi Yancong. et al. Research on vehicle speed measurement by video image based on tsai's two stage method.The 5th International Conference on Computer Science \& Education, Hefei, China. August 24-27, 2010

[5] GHOSH, S. K.; Image motion compensation through augmented collinearity equations, Optical Engineering; v. 24, n.6, 1985, p. 1014-1017.

[6] TSAI. R. Y. A versatile camera calibration technique for high-accuracy 3D machine vision metrology using off-the-shelf TV cameras andlenses.IEEE Journal of Robotics and Automation, RA-3(4), August1987:323344.

[7] BRADSKI, G; KAEHLER, A.Learning OpenCV: computer vision with the OpenCV library. O'Reilly Press, Cambridge, MA, 2008.

[8] PETRIE, G; EL NIWERI, A.E.H. The applicability of space imagery to the small scale topographic mapping of developing countries: a case study - the Sudan. ISPRS Journal of Photogrammetry and Remote Sensing, v. 47, n. 1, p. 1-42, 1992.

[9] NOVAK, K. Rectification of digital imagery. Photogrammetric Engineering and Remote Sensing, v. 58, n. 3, p. 339-344, 1992. 